\title{
Ethical, legal and professional accountability in emergency nursing practice: an ethnographic observational study.
}

\author{
Alfonso Rubio-Navarro, PhD, MSc, RN ${ }^{\mathrm{a}}$ \\ Diego Jose Garcia-Capilla, PhD, MSc ${ }^{\text {b }}$ \\ María Jose Torralba-Madrid, PhD, MSc, RN c \\ Jane Rutty, PhD, MSc, BSc(Hons) DPSN RN ${ }^{\mathrm{d}}$
}

\section{Corresponding author:}

Alfonso Rubio Navarro

Email: alfonso.rubio.navarro@gmail.com

Telephone number: 07926642498

${ }^{a}$ Emergency Department, University Hospitals of Leicester NHS Trust, Leicester, LE1 5WW, United Kingdom. Email: alfonso.rubio.navarro@gmail.com

${ }^{\mathrm{b}}$ Philosophy Faculty, University of Murcia, Campus Universitario Street, 30100, Murcia, Spain.Email: djgarcia@um.es

c Nursing Faculty, University of Murcia, Campus Universitario Street, 30100, Murcia, Spain.Email: mjtorral@um.es

${ }^{d}$ Faculty of Health and Life Sciences, De Montfort University, LE1 9BH, Leicester, United Kingdom. Email: jrutty@dmu.ac.uk

International Emergency Nursing 2019 
Conflict of Interest statement: None declared.

Ethical statement: As part of the research study "Ethical and legal accountability in nursing clinical practice: analysis of protocols and clinical activity in an English emergency department”, it had the approval of the National Health Service Health Research Authority, University Hospitals of Leicester Research and Innovation Department and De Montfort University Faculty of Health and Life Sciences Research Ethics Committee.

Funding: This research did not receive any specific grant from funding agencies in the public, commercial, or not-for-profit sectors. 


\begin{abstract}
Introduction

Accountability in nursing practice is a concept that influences quality care, decisionmaking, safety standards and staff values. Therefore, understanding accountability and how it affects nursing practice could improve patient care and nurses’ working conditions.
\end{abstract}

\title{
Aim
}

The aim of this study was to find factors that influenced ethical, legal and professional accountability in emergency nursing practice.

\section{Methods}

A qualitative ethnographic approach using participant observation through convenience sampling was employed as the data collection method, while ethnographic content analysis was used for data analysis.

\section{Results}

The factors linked to nursing accountability found were classified into four main themes: daily dynamics, work environment evolution, customs and routines and bioethics principles’ application.

\section{Discussion}

The long-term effect of chronic high workload and crowding, which affects nursing accountability, could promote burnout in a junior ED workforce. Changes in the nurses’ working conditions need to be implemented to limit the workload to which an ED nurse is subjected to. 


\section{Conclusion}

ED nurses have to manage their accountability in difficult situations regularly, which followed patterns of four main themes across the majority of situations. Nonetheless, all those factors were influenced by nursing workload, an ever-present factor that was always considered by ED nurses during decision-making.

\section{Keywords}

Accountability; clinical ethics; decision-making; emergency nursing; legal; motivation; nurse-patient relationship; professional ethics; work conditions. 


\section{Introduction}

Accountability in clinical nursing practice is a concept linked to both legal and ethical aspects of practice and influences quality care, decision-making, safety standards and staff values. Therefore, understanding accountability and how it affects Emergency Department (ED) nursing practice could improve emergency patient care and ED nurses’ working conditions. However, even though accountability has a profound effect on how care is provided, current literature in the specific area of emergency care is very limited [1].

Nursing practice in an English ED has several characteristics that are unique to that role. ED nurses have to manage a virtually unlimited number of acute patients with limited resources while maintaining a complex work-life balance [2]. Moreover, the recent rise of people attending EDs across England has increased the workload of ED nurses, worsening care quality, patient safety and nurses’ working conditions. However, all the factors are regulated by nursing accountability [3].

Accountability is defined by Lewis and Batey as: "the fulfilment of a formal obligation to disclose to relevant others the purposes, principles, procedures, relationships, results, income and expenditures for which you have authority” [4]. Depending on the type of formal obligation, there are three accountability domains: legal, based on law; professional, based on deontological codes; and ethical, based on moral principles and values.

Regarding recent articles, only a small number can be linked to ED nursing accountability. Langeland and Sørlie considered the existence of vulnerability when facing ethical dilemmas in an ED in Norway [5], while Ebben et al. indicated the factors 
involved in monitoring a national ED protocol in the Netherlands [6]. These articles refer to common issues that are present in most British EDs, like vulnerable patients or multidisciplinary practice cohesion.

The use of ethics in accountability issues inside an ED has already been utilised in other countries such as Taiwan, as shown by Lin et al [7]. Therefore, if there was more research evidence on factors that affect nursing accountability we could influence accountability positively and promote safe and holistic care in clinical practice.

\section{Aim}

The aim of this study was to find factors that influenced ethical, legal and professional accountability in emergency nursing practice.

\section{Methods}

\section{Study design}

Since 2016, revalidation in the UK is part of a process that all nurses need to follow to maintain their registration with the Nursing and Midwifery Council (NMC). Revalidation is intended to assist nurses in demonstrating safe and effective practice. This includes reflecting on their personal practice so as to demonstrate that they are following the standards set by the NMC Code [8].

Therefore, we kept a personal reflective journal, generating reflective accounts to explain what we had learnt from experience in an English ED. No identifiable information from patients or staff was included within these reflections in line with patient confidentiality and data protection policies. 
After revising the reflective journal, we decided to research matters surrounding ethical, legal and professional nursing accountability in the ED more formally with a team of a Nursing professor, a Philosophy professor and a $\mathrm{PhD}$ student that also worked as a nurse in that ED and performed the data collection. We subsequently employed an ethnographic approach using participant observation as the data collection method, convenience sampling and ethnographic content analysis to analyse the collected data.

\section{Data collection}

\section{Setting}

The naturalistic setting of ED offered a number of advantages. Some of those were having first-hand experience in the setting and with its individuals, the ability to see things that individuals in the setting might routinely not notice and the opportunity to learn things that participants may not be willing to share in an interview.

\section{Observation}

Observation was undertaken from May 2017 to May 2018 while still being involved in all routine ED activities as a registered nurse. The research role as "complete participant" as described by DeWalt (the researcher is integrated into the community of study) [9] was made known to all participants. Data were recorded in a field diary and transcribed daily into Nvivo for further analysis.

The observational period involved three steps as described by Angrossino and Mays de Perez [10]:

1. Descriptive observation (all preconceptions were eliminated and nothing was taken for granted); 
2. Focused observation (sole observation of what was pertinent to the issue at hand);

3. Selective observation (focused on specific forms of a more general category).

The aim of utilising observational data as "complete participant" was to understand emergency nurses' environment from an insider's perspective, discovering "the hows and whys of human behaviour in a particular context” [11]. Sharing those experiences enabled the recording of important nursing practice elements that were only visible to those that were present [11]. All data were collected by the same researcher.

\section{Sampling}

The inclusion criteria were registered nurses that worked in that ED during that shift and were able to give their consent. The exclusion criteria were non-registered nurses, nurses that were not able to give their consent or nurses that could not be informed extensively about this research (e.g. non-regular agency nurses). The limited number of sample criteria and the use of convenience sampling was aimed to: increase recruitment; avoid skewing the sample’s diversity and boost data depth, complexity and reliability [12].

Sampling stopped when there was enough information to replicate the study and no new information was obtained, therefore additional coding was not necessary. This met Fusch and Ness' definition of data saturation [13].

\section{Data analysis}

Data analysis utilised an ethnographic content analysis method [14]. During the participant observation phase, a running record of themes was kept to enable exploration and to facilitate future observations to become more focused and directed. 
Ethnographic content analysis allowed the contextualisation of human action in relation to their environment through fieldwork and supported the counting and classification of concepts into themes, providing descriptive information of them. This method included six phases: (1) coding frame creation, (2) field diary transcript division, (3) first pilot phase, (4) second pilot phase, (5) first main coding phase and (6) second main coding phase [14].

Themes and sub-themes generated from field observations and field diary transcripts were then organised into a coding frame using descriptive coding and subcoding [15] (phase 1). Transcripts were divided as units of coding based on a thematic criterion (phase 2), from which a random selection of $25 \%$ of them were used in two pilot phases 13 days apart (phases 3 and 4). After evaluating both pilot phases using Schreier's coding frame requisites (unidimensionality, mutual exclusiveness, exhaustiveness, saturation, reliability and validity) [16], the coding frame was tested and utilised in the first and second main coding phases, when all transcripts were coded twice 11 days apart (phases 5 and 6). This enabled the results to be compared and reliable themes to be created, again meeting Schreier’s requirements [16].

All transcripts were then transferred to Nvivo (version 11.4.1.1064) to aid codification. However, no automated computerised methods were used to code data.

\section{Ethical considerations}

This research study had the approval of: the National Health Service Health Research Authority; University Hospitals of Leicester Research and Innovation Department and; De Montfort University Faculty of Health and Life Sciences Research Ethics Committee. 
Informed consent for this study included three key components: communication of information, comprehension of information, and voluntary participation. The ethical committees waivered the need for individual written informed consent due to the minimal risk of the research. However, in order to obtain oral consent, we began by informing potential participants in both writing and verbally of the purposes and procedures of the research, the risks and benefits associated with the study, the right for participant withdrawal at any time without penalty and how the data provided by the participant would be protected and stored to protect confidentiality. Contact information was also provided to participants prior to the start of the study should anyone have concerns about any aspect of the study along with who to complain to if something should go wrong.

As an added measure, participants were reminded again about the research through posters displayed in the ED Staff Room and during nursing clinical handovers, giving them the opportunity to withdraw their consent and/or to have their contributions withdrawn from the data collected to date.

\section{Trustworthiness}

This research used Korstjent and Moser's definition of trustworthiness standards [17]. Prolonged and persistent engagement were used to boost credibility, while thick description of the methodology and results and the presentation of the whole field diary through a public repository improved transferability [18].

Dependability and confirmability were ascertained following the Standards for Reporting Qualitative Research (SRQR) checklist [19] and through a transparent description of the research steps taken. Furthermore, the role of the researcher in ED was considered in the field diary and documented in the discussion, which enhanced reflexivity. 


\section{Results}

\section{Demographics}

One hundred and eighty-six nurses were observed during 146 observations periods, making a total of 1870 hours of data collection. No nurse requested to have their consent withdrawn from this study. The average participant was a British female adult registered nurse, but the sample overall was diverse (see table 1).

\section{Findings}

The factors linked to nursing accountability found were classified into four main themes: daily dynamics, work environment evolution, customs and routines and bioethics principles' applications. Each of them is further divided into separate sub-themes (see table 2). Observed quotes are identified with quotation marks.

\section{Daily dynamics}

A standard shift was categorised by a moderate, continuous and constant flow of patients through the department. It started with the distribution of nurses and healthcare assistants (HCAs) onto the different areas in ED, where each nurse then received handover from their colleagues on the previous shift.

Patients followed an established journey through ED depending on their illness and its acuity, which was led by a nurse coordinator in each area and the nurse in charge (NIC) for the whole department. Nurse coordinators also allocated the order of breaks, ensuring that every nurse was able to rest for 30 minutes every six hours. 
When the nursing shift was close to finishing, nurses and HCAs replenished the stock of every area to allow a continuous provision of care. Lastly, each nurse gave a handover of their patients to the allocated nurse for the next shift, maintaining a named nurse for each patient at all times.

During shifts with increased patient inflow and increased patient outflow the bottleneck in the assessment bay forced the NIC to accelerate the distribution of patients across the department and the hospital. However, the increasing patient inflow and the lack of nursing staff could not be supported by the hospital long-term, so this shift evolved into shifts with increased patient inflow and reduced patient outflow, the most common shift during the winter months.

"I cannot cope with everything anymore, I used to years ago but now there are so many patients and so little time"

The lack of human resources and hospital beds that defined that shift affected every step in a predictable way. Patients were redistributed into less crowded non-acute areas, while the NIC allocated more patients per nurse, forcing nurses to work faster and less safely. Safety standards were laxer, allowing patients to be treated in corridors or inside ambulances. Breaks were allocated as normal, but junior nurses tended to go later to their breaks or refused them to have more time to attend their patients.

“I struggle to go to my break sometimes, I don’t know what I'll find when I come back" Under the pressure of crowding and high patient inflow, nursing care provision branched into two generalised trends: nurses that prioritised efficiency over safety, making hazardous decisions to be able to care for more patients; and nurses that ranked safety over efficiency, choosing to overlook the needs of some patients in favour of other 
patients or their own. Nurses did not make this decision willingly, but the lack of resources forced them to choose.

“The main problems are always beds and staffing, if we had that we could deliver terrific care and not feel so bad"

\section{$\underline{\text { Work environment evolution }}$}

The constant struggle of EDs is to adapt to the needs of an ever-growing population, which results in constant evolution. The resulting changes could be classified into three sub-themes: nursing staff turnover, material resource distribution and work dynamics.

The constant nursing staff turnover was a recurrent problem in ED. This phenomenon was distributed throughout the year, although it was in the winter season when most nurses were planning to leave, mainly motivated by the winter workload and erratic shifts. This fact increased the use of agency nurses and pushed junior nurses to take senior roles for which they were not prepared for, hindering the leadership of the department and encouraging defensive practice.

"It's the same every year: experienced nurses go and newly-qualified arrive lost and scared”

Another element that changed consistently was the department's layout (material resource distribution), which accommodated more patients with the same number of nurses. Due to those changes, the work dynamics changed accordingly. New practices and policies were introduced constantly without appropriate training, obliging nurses to adapt their practice to new rules repeatedly while providing safe and documented care. 
The broad result of this process of constant unplanned evolution was irregular work dynamics, since the nursing team did not know their colleagues, their department or which rules to follow, as shown by their interactions with the researcher. This situation encouraged them to focus on the basics such as basic care provision, their code of conduct or their personal values.

“Since I am part-time, every day feels like the first one in this place with so many changes happening all the time”

\section{$\underline{\text { Customs and routines }}$}

Clinical practice has been led by a series of rituals that characterises it (customs and routines), some forged through custom and tradition, others implemented in the pursuit of efficiency. Several of these rituals reflected decisions that nurses had made during their clinical practice and how they used different ethical, legal, professional and personal factors to create a solid foundation to justify their reasoning.

Different handovers and transfers were necessary to maintain continuity of care between shifts, areas and departments. Temporal handovers during breaks were a constant issue, since the nurse that received the handover doubled their patients for a period of time. Consequently, they mostly prioritised their patients and overlooked their colleagues' patients, even if in theory they were accountable for both groups. This created an unsafe workload imbalance that was widely accepted by both healthcare professionals and managers.

"So many transfers today, I felt like the only nurse in majors, I barely managed my own patients” 
In relation to nursing care, it involved performing techniques (technical competencies) that were guided by specific policies. However, nurses interpreted those policies on some occasions to be able to provide emergency care for more patients, even if that decision could facilitate a possible clinical error. The main example of this phenomenon was the administration of intravenous medication, since nurses did not always verify the drug as the local policy suggested to be able to administer more life-saving medication in the same amount of time. It is important to acknowledge that all such incidents were reported in line with local risk management systems to support not only learning, but patient care improvement.

ED nurses worked in multidisciplinary teams, so the relationships between nurses and other healthcare professionals influenced their accountability. There were several examples of this phenomenon: task delegation to HCAs, shared accountability with other nurses, overruled decision by managers, supervision of apprentices in practice, etc. Even if these situations were different in many ways, they shared a common phenomenon: the influence of nurses in the decisions made by them or others was not exclusively linked to their role but to the other person's perception of the nurse. This was shaped by factors such as the nurse's knowledge, charisma or decision-making skills.

"I'll do what she tells me to do, I trust her more than [my manager]"

Each nurse-patient relationship was unique, but they followed various customs and preconceived ideas. The most prevalent ones were the stigmas linked to patients with mental health or social needs, which devalued their care in favour of patients with physical needs, independently of their acuity. Those ideas promoted an ineffective nursepatient relationship, in which the nurse and the patient had different perspectives on how care should be provided and under what circumstances. 
“Again? He’s always here. Wake him up, let's see what he wants”

\section{Bioethics principles’ application}

ED nurses are aware that nonmaleficence is a fundamental principle to be applied in their daily practice. However, nurses could not always avoid patients suffering physical, mental and emotional deterioration. This behaviour was widespread among ED nurses for two main reasons: the perception of the patient as their responsibility, since they mainly cared for patients allocated to them, and the dehumanisation of care, a common phenomenon in crowded EDs. These behaviours were used by some nurses to rationalise their guilt for not being able to provide care for everyone, since they argued that they had offered the best care possible under dire circumstances.

“I’ve seen this every day for many many years, mate. It doesn't affect me anymore, I do what I can and I go home”

The continuous workload increment facilitated the proliferation of defensive practice, in which some nurses performed the minimal care required by hospital policies and applicable regulations to devote a considerable percentage of their time to documentation. Nurses who practised defensively usually argued that they will not risk their professional registration and their way of life to benefit a patient who did not appreciate the care they received.

“If you don’t protect yourself and document properly, you'll lose your PIN and the patient will not [care] about you”

In relation to autonomy, some elderly patients who were treated in ED indicated their displeasure when they realised they would be admitted into hospital, arguing that every time they spent a period of time in bed they lost some of their independence. However, 
some adult patients wanted to be admitted for reasons relatively easy to treat in the community, but they felt more secure receiving care at the hospital. This is the self-care paradox, in which patients unable to meet their basic needs rejected professional support while independent patients demanded that others covered needs that they could cover by themselves.

"It is such a shame that she did not call 999 earlier. She keeps saying that she doesn't want to be a nuisance”

The NIC was able to modify the distribution of personnel in real time to adapt to the department's situation. Nevertheless, the distribution of human resources by the NIC was subjective, depending on the objectives’ prioritisation order (e.g. patient flow, quality of care, patient safety, staff safety, fines and complaints avoidance). Each NIC pursued different goals in different situations, but the prevalence of patient flow and the avoidance of fines and complaints on top of other objectives were observed on most NICs.

“They opened the corridor again with just one patient waiting in the ambulances, what are they thinking?”

\section{Discussion}

During periods of high workload nurses had to modify their practice to adapt to the situation. However, although these busy periods were constant, nurses were accustomed to bending policies and managing unsafe workloads, as they are accountable to every eventuality that affected their patients. These risks were already found by Kirk and Nilsen and Adriaenssens et al., who indicated that nurses would not follow policies strictly if believed that they affected patient flow and confirming the relationship between inappropriate levels of nursing staff and lower quality of care [20,21]. Moreover, in this 
research nurses also felt accountable for their continuous development to follow work environment evolution, but the constant changes and the lack of support hindered their efficiency even more. Considering that there is peer-reviewed literature supporting the link between higher workload and lower quality of care, it is likely that this phenomenon can be extrapolated to other EDs.

The customs and techniques shown by ED nurses were a result of the aforementioned constant high workload. They prioritised speed and acuteness to be able to provide minimal care, so they did not have time to follow policies or establish effective working relationships. This rushed mentality that has been engrained into ED nurses, according to Wolf et al., leads to destructive relationships between ED healthcare professionals affecting patient care, nursing culture and staff retention [22]. It can be argued that nurses work quickly to care for all their patients, but the detrimental effects that Wolf et al. described are not only linked to rushed care but also to lower standards that come from always providing minimal care and seen it as the norm.

From not being able to alleviate suffering to the limiting nature of human resources, the challenges linked with Bioethics’ principles are created as a response for not having enough resources to provide care for everyone. Nonetheless, nurses felt accountable for not being able to solve those challenges on their own, which put a profound burden on them that can increase burnout. Wolf et al. called this burden "moral distress", an environment driven phenomenon [23] that afflict nurses professionally and personally. We agree with Wolf et al. that the lack a validated instrument to measure moral distress hinders the ability of institutions to tackle it, so it should be a priority for future research.

Conversely, there are two factors that influenced nursing accountability and are linked with all four themes: high workload and crowding. According to Carter et al., crowding 
is linked to negative outcomes in ED and high nursing workload increases medication errors and associated infections [24], but they also have a harmful effect in nursing accountability. This negative effect is based on the fact that the nurse is accountable for the patient's care but does not have the resources necessary to care for them, which creates a complex ethical dilemma. This ethical conundrum that ED nurses face daily further increases moral distress, since even if they have the knowledge and ability to provide high quality care they are unable to do so due to external factors out of their control, conflicting their values and eroding the reason why they became nurses: to care.

The long-term effect of chronic high workload and crowding in nursing accountability could create an ED junior burnt out workforce, since other nurses could find better working conditions and satisfaction elsewhere. Specific recommendations include healthcare policies and changes in nursing working conditions being implemented to limit the workload to which an ED nurse is subjected to. These measures would reduce accountability issues like clinical errors, lack of basic technical competencies within the team or relationship dissonance between the patient and the nurse. These changes would promote safer and more efficient care, since the nurse would feel confident in their practice and protected in their role as the patient's advocate and carer. Furthermore, if nurses working in EDs feel that their accountability is not a handicap for their practice they will feel less stressed, raising staff retention and satisfaction.

\section{Strengths and limitations}

The observation period allowed the analysis of nursing accountability through an ED's evolution and provided large amounts of data, facilitating the ability to discard anecdotic evidence to find patterns in nursing behaviour while following rigorous trustworthiness standards. Moreover, this research study considered nursing accountability holistically, 
not only in its legal variant but also its ethical and professional perspectives through nursing values and deontological codes.

On the other hand, there were some limitations to this study, which were minimised by the research methodology. It can be argued that when the researcher practised as a complete participant observer alongside other nurses there was a possibility to influence the practice that they wanted to analyse. It should be borne in mind that all nurses interact during practice and affect each other, so since the researcher was immersed in the department as an equal nurse their effect on the nurses was different than the effect that any other nurse may have. Additionally, the interaction with other nurses provided key information that would be very difficult to obtain through non-participant observation (e.g. professional relationships within the multidisciplinary team).

Moreover, it can be argued that the information obtained could be biased, since it has been obtained and analysed through one individual. However, complete participant observation is a recognised technique that relies upon the researcher's experience for portraying the behaviour of the community they live with. This experience was also heavily complemented with hundreds of informal conversation with other nurses, but those conversations were not recorded literally to avoid confidentiality and ethical problems and so as to not interrupt clinical practice during an extended amount of time.

A separate reflective journal was maintained to identify any assumptions or preconceptions through self-reflection and to avoid those which could skew the data. Moreover, we were aware of how the researcher relationship could modify the participants' behaviour, so we maintained a cordial but neutral approach to them. 


\section{Conclusion}

ED nurses have to manage their accountability in difficult situations regularly, for which they make decisions based on different ethical, legal and professional factors. However, there were patterns that showed four main themes across the majority of situations.

Factors like staff interactions, care provision or technical competencies followed a pattern that could be predicted. Nonetheless, all those factors were influenced by nursing workload, an ever-present factor that was always considered by ED nurses during decision-making. The effect of nursing workload in their accountability was holistic, since it affected how they interpreted regulations, codes of conduct and their values when making decisions linked to the patients they were accountable for. Further research is needed to understand the long-term effect of high workload on nursing accountability, both in regards to their practice and their values. 


\section{References}

[1] Johnstone M-J. Bioethics : a nursing perspective. 6th ed. Elsevier; 2015.

[2] Person J, Spiva L, Hart P. The culture of an emergency department: An ethnographic study. Int Emerg Nurs 2013;21:222-7. doi:10.1016/J.IENJ.2012.10.001.

[3] National Audit Office. Reducing emergency admissions. 2018.

[4] Griffith R, Tengnah C. Law and professional issues in nursing. Third. SAGE; 2014.

[5] Langeland K, Sørlie V. Ethical challenges in nursing emergency practice. J Clin Nurs 2011;20:2064-70. doi:10.1111/j.1365-2702.2010.03606.x.

[6] Ebben RHA, Vloet LCM, de Groot JM, van Achterberg T. Factors influencing adherence to an emergency department national protocol. Eur J Emerg Med 2012;19:53-6. doi:10.1097/MEJ.0b013e3283474a87.

[7] Lin Y-K, Lee W-C, Kuo L-C, Cheng Y-C, Lin C-J, Lin H-L, et al. Building an ethical environment improves patient privacy and satisfaction in the crowded emergency department: a quasi-experimental study. BMC Med Ethics 2013;14:8. doi:10.1186/1472-6939-14-8.

[8] Nursing and Midwifery Council. Revalidation 2018. http://revalidation.nmc.org.uk/ (accessed July 17, 2018).

[9] DeWalt KM, DeWalt BR. Participant observation: a guide for fieldworkers. Rowman \& Littlefield; 2011. 
[10] Angrossino M, Mays de Perez K. Rethinking observation: from method to context. In: Denzin N, Lincoln Y, editors. Collect. Interpret. Qual. Mater. 2nd ed., London: Sage Publications; 2003.

[11] Guest G, Namey EE, Mitchell ML. Collecting Qualitative Data: A Field Manual for Applied Research. London: SAGE Publications, Ltd; 2013. doi:10.4135/9781506374680.

[12] Etikan I, Musa SA, Alkassim RS. Comparison of Convenience Sampling and Purposive Sampling. Am J Theor Appl Stat 2016;5:1. doi:10.11648/j.ajtas.20160501.11.

[13] Fusch P, Ness L. Are We There Yet? Data Saturation in Qualitative Research. Qual Rep 2015;20.

[14] Altheide DL. Reflections: Ethnographic content analysis. Qual Sociol 1987;10:65_ 77. doi:10.1007/BF00988269.

[15] Saldaña J. The coding manual for qualitative researchers. 2nd ed. London: Sage Publications; 2013.

[16] Schreier M. Qualitative content analysis in practice. 1st ed. London: Sage Publications; 2012.

[17] Korstjens I, Moser A. European Journal of General Practice Series: Practical guidance to qualitative research. Part 4: Trustworthiness and publishing. Eur J Gen Pract 2018;24:120-4. doi:10.1080/13814788.2017.1375092org/10.1080/13814788.2017.1375092.

[18] Blinded for peer review 
[19] O’Brien BC, Harris IB, Beckman TJ, Reed DA, Cook DA. Standards for reporting qualitative research: a synthesis of recommendations. Acad Med 2014;89:124551. doi:10.1097/ACM.0000000000000388.

[20] Adriaenssens J, De Gucht V, Maes S. Determinants and prevalence of burnout in emergency nurses: A systematic review of 25 years of research. Int J Nurs Stud 2015;52:649-61. doi:10.1016/J.IJNURSTU.2014.11.004.

[21] Kirk JW, Nilsen P. Implementing evidence-based practices in an emergency department: contradictions exposed when prioritising a flow culture. J Clin Nurs 2016;25:555-65. doi:10.1111/jocn.13092.

[22] Wolf LA, Perhats C, Clark PR, Moon MD, Zavotsky KE. Workplace bullying in emergency nursing: Development of a grounded theory using situational analysis. Int Emerg Nurs 2018;39:33-9. doi:10.1016/J.IENJ.2017.09.002.

[23] Wolf LA, Perhats C, Delao AM, Moon MD, Clark PR, Zavotsky KE. "It’s a Burden You Carry”: Describing Moral Distress in Emergency Nursing. J Emerg Nurs 2016;42:37-46. doi:10.1016/j.jen.2015.08.008.

[24] Carter EJ, Pouch SM, Larson EL. The Relationship Between Emergency Department Crowding and Patient Outcomes: A Systematic Review. J Nurs Scholarsh 2014;46:106-15. doi:10.1111/jnu.12055. 
Table 1 - Participants’ demographics

\begin{tabular}{|c|c|}
\hline Gender & $\begin{array}{l}\text { - } 153 \text { women }(82.2 \%) \\
\text { - } 33 \text { men }(17.8 \%)\end{array}$ \\
\hline $\begin{array}{l}\text { Years of experience (full time equivalent) } \\
\text { as a registered nurse, divided by juniors } \\
\text { (less than } 2 \text { years) or seniors (more than } \\
2 \text { years) }\end{array}$ & $\begin{array}{l}\text { - } \quad 79 \text { junior nurses }(42.5 \%) \\
\text { - } \quad 107 \text { senior nurses }(57.5 \%)\end{array}$ \\
\hline Nursing grade & $\begin{array}{l}\text { - } 145 \text { staff nurses ( } 78 \%) \\
\text { - } 27 \text { deputy sisters / charge nurses } \\
(14.7 \%) \\
\text { - } 14 \text { sisters / charge nurses }(7.3 \%)\end{array}$ \\
\hline Cultural origin and nationality & $\begin{array}{l}\text { - } 145 \text { British nurses } \\
0 \quad 117 \text { White British nurses } \\
(63 \%) \\
0 \quad 28 \text { Non-White British nurses } \\
(15 \%) \\
\text { - } 41 \text { Non- British nurses } \\
0 \quad 21 \text { European nurses (11.3\%) } \\
0 \quad 20 \text { Non-European nurses } \\
(10.7 \%)\end{array}$ \\
\hline Type of registration & $\begin{array}{ll}-162 \text { adult nurses }(87.1 \%) \\
\text { - } \quad 24 \text { children's nurses ( } 12.9 \%)\end{array}$ \\
\hline
\end{tabular}




\begin{tabular}{|c|c|}
\hline Main themes & $\begin{array}{l}\text { Sub-themes (derivative from a main theme) } \\
\text { Participants' quotes between quotation marks }\end{array}$ \\
\hline \multirow[t]{6}{*}{$\begin{array}{l}\text { Daily dynamics } \\
\text { (recurrent nursing practice structure that } \\
\text { repeats following an specific pattern } \\
\text { dependant on patient flow) }\end{array}$} & $\begin{array}{l}\text { First handover and personnel distribution } \\
\text { "We work in different areas each day, but when you can triage you } \\
\text { only do that and it gets repetitive" } \\
\text { "When the handover first time in the morning is rubbish, it's so } \\
\text { much difficult" }\end{array}$ \\
\hline & $\begin{array}{l}\text { Patient distribution } \\
\text { "Patients that are red called come to resus as a priority; they can } \\
\text { be distributed to other areas if they aren't sick" }\end{array}$ \\
\hline & $\begin{array}{l}\text { Responsibility and leadership interactions } \\
\text { "I feel that I am a buffer between junior nurses and managers, we } \\
\text { just follow different objectives" }\end{array}$ \\
\hline & $\begin{array}{l}\text { Shift breaks' arrangement } \\
\text { "I struggle to go to my break sometimes, I don't know what I'll find } \\
\text { when I come back" }\end{array}$ \\
\hline & $\begin{array}{l}\text { Nursing care provision } \\
\text { "The main problems are always beds and staffing, if we had that } \\
\text { we could deliver terrific care and not feel so bad" } \\
\text { "I cannot cope with everything anymore, I used to years ago but } \\
\text { now there are so many patients and so little time" }\end{array}$ \\
\hline & $\begin{array}{l}\text { Checklists and last handover } \\
\text { "Checklists are a matter of safety, you don't want to be in a cardiac } \\
\text { arrest without equipment" }\end{array}$ \\
\hline \multirow{2}{*}{$\begin{array}{l}\text { Work environment evolution } \\
\text { (progressive changes of nursing staff, } \\
\text { practice and resources over a period of } \\
\text { time) }\end{array}$} & $\begin{array}{l}\text { Nursing staff turnover } \\
\text { "It's the same every year: experienced nurses go and newly- } \\
\text { qualified arrive lost and scared" }\end{array}$ \\
\hline & $\begin{array}{l}\text { Material resource distribution } \\
\text { "Do you know the new } 3 \text { policies that we supposed to apply in } \\
\text { assessment bay? Me neither" }\end{array}$ \\
\hline
\end{tabular}




\begin{tabular}{|c|c|}
\hline & $\begin{array}{l}\text { Work dynamics } \\
\text { "Since I am part-time, every day feels like the first one in this place } \\
\text { with so many changes happening all the time" }\end{array}$ \\
\hline \multirow[t]{4}{*}{$\begin{array}{l}\text { Customs and routines } \\
\text { (periodic rituals that affect nursing practice } \\
\text { but do not follow a predictable pattern) }\end{array}$} & $\begin{array}{l}\text { Patient handover and transfer } \\
\text { "So many transfers today, I felt like the only nurse in majors, I } \\
\text { barely managed my own patients" }\end{array}$ \\
\hline & $\begin{array}{l}\text { Technical competencies } \\
\text { "I was stuck in the treatment room for } 15 \text { minutes checking drugs } \\
\text { for everyone, everyone is septic today" }\end{array}$ \\
\hline & $\begin{array}{l}\text { Relationships between nurses and other } \\
\text { healthcare professionals } \\
\text { "I'll do what she tells me to do, I trust her more than [my manager]" }\end{array}$ \\
\hline & $\begin{array}{l}\text { Nurse-patient relationship } \\
\text { “Again? He's always here. Wake him up, let's see what he wants" }\end{array}$ \\
\hline \multirow[t]{4}{*}{$\begin{array}{l}\text { Bioethics principles' application } \\
\text { ( use of bioethics' principles to explain the } \\
\text { nurses' respond to ethical challenges) }\end{array}$} & $\begin{array}{l}\text { Nonmaleficence and tolerance to others' suffering } \\
\text { "I've seen this every day for many many years, mate. It doesn't } \\
\text { affect me anymore, I do what I can and I go home" }\end{array}$ \\
\hline & $\begin{array}{l}\text { Beneficence and defensive practice } \\
\text { "If you don't protect yourself and document properly, you'll lose } \\
\text { your PIN and the patient will not [care] about you" }\end{array}$ \\
\hline & $\begin{array}{l}\text { Autonomy and the self-care paradox } \\
\text { "What does he want from me? His assessment is clear but he } \\
\text { refuses to leave without being admitted" } \\
\text { "It is such a shame that she did not call } 999 \text { earlier. She keeps saying } \\
\text { that she doesn't want to be a nuisance" }\end{array}$ \\
\hline & $\begin{array}{l}\text { Justice and subjective distribution of human } \\
\text { resources } \\
\text { "They opened the corridor again with just one patient waiting in } \\
\text { the ambulances, what are they thinking?" }\end{array}$ \\
\hline
\end{tabular}

